\begin{tabular}{|c|l|}
\hline Title & Non-invasive measurement of floating-sinking motion of a large object in a gas solid fluidized bed \\
\hline Author(s) & $\begin{array}{l}\text { Yoshimori, Wataru; Ikegai, Tomoki; Uemoto, Koshi; Narita, Shohei; Harada, Shusaku; Oshitani, Jun; T suji, Takuy a; } \\
\text { Kaiwara, Hirokazu; Matsuoka, Kei }\end{array}$ \\
\hline Citation & $\begin{array}{l}\text { Granular matter, 21/3), 42 } \\
\text { https://doi.org/L0.1007/310035-019-0897-3 }\end{array}$ \\
\hline Issue Date & 2019-08 \\
\hline Doc URL & http://hdl.handle.net/2115//8998 \\
\hline Rights & The final publication is available at link.springer.com \\
\hline Type & article (author version) \\
\hline File Information & harada6.pdf \\
\hline
\end{tabular}

Instructions for use 


\title{
Non-Invasive Measurement of Floating-Sinking Motion of a Large Object in a Gas-Solid Fluidized Bed
}

Wataru Yoshimori, Tomoki Ikegai, Koshi Uemoto, Shohei Narita, and Shusaku Harada* Faculty of Engineering, Hokkaido University, Sapporo, Japan, 060-8628

\author{
Jun Oshitani
}

Faculty of Engineering, Okayama University of Science, Okayama, Japan, 700-0005

\section{Takuya Tsuji}

Graduate School of Engineering, Osaka University, Suita, Japan, 565-0871

Hirokazu Kajiwara

Ebara Environmental Plant Co., LTD., Tokyo, Japan, 144-0042

Kei Matsuoka

Ebara Corporation, Tokyo, Japan, 144-8510

(Dated: June 26, 2019) 


\begin{abstract}
A Lagrangian sensor system has been established to non-invasively measure both the vertical position and dynamic force acting on itself. It consists of a 3 -axis acceleration sensor, a 3-axis magnetometer, a microcontroller, a wireless module, batteries, and external electromagnetic coils. In this study, we applied the system to a free-moving coarse object in a gas-solid fluidized bed. The floating and sinking motions of the object in the fluidized bed are essentially caused by differences between its density and the apparent density of the fluidized media. However, the object sometimes shows strange behavior under the influence of variance in the fluidization state. We measured the temporal change of the upward force acting on the object as well as the vertical position, which is invisible from the outside. The experimental results indicate that the force acting on the object differs significantly between the floating and sinking states and is greatly complicated by interference with rising bubbles in the fluidized bed. The probability density of the vertical position of the object shows that its motion is explained not only by hydrostatic effects, but also by inhomogeneity of the fluidization state in the bed.
\end{abstract}

PACS numbers: 47.61.Jd

*Electronic address: harada@eng.hokudai.ac.jp 


\section{INTRODUCTION}

Multiphase flow with solid particles, i.e., liquid-solid flow or gas-solid flow, is known to be an unpredictable system although it has many applications in various engineering fields. In these flows, particles change their motion under the influence of the surrounding flow, while the fluid flow changes the velocity and pressure field according to the particle motion. These complicated interactions are essentially momentum exchange at the boundary between phases and it constitutes a barrier to understanding multiphase flow systems.

The particle-fluid interaction in multiphase flow has been investigated theoretically, experimentally, and numerically [1]. Generally, it has been considered as a fluid force acting on solid particles. For example, numerous studies on the drag and lift forces acting on particles have been performed [2]. However, most of these studies have been conducted under the assumption of a fixed particle in a steady flow or a moving particle in a static fluid with constant velocity. In realistic cases, the particle changes its motion at every moment in the unsteady flow field. It is known that unsteady particle and fluid motions bring about additional fluid forces such as added mass and history forces, which are functions of relative acceleration, and they are significant particularly in liquid-solid flows [1, 2]. Moreover, the particle-particle interaction (collision or hydrodynamic interaction) complicates the whole flow field. Therefore most studies assuming steady flow or fixed particles are inadequate for understanding practical multiphase flows.

When we want to know the force acting on a freely moving solid object, one possible approach is to measure the trajectory (position) of the object and then calculate instantaneous force (acceleration) from the second derivatives of temporal position data. However, the problem with this approach is that, in general, a large dispersed error occurs in the process of differentiation of discrete data. On the other hand, the calculation of the position from discrete acceleration data of a fleely moving object produces a large cumulative error via an integration process. Therefore, if we want to know the force acting on the object, the instantaneous acceleration should be measured directly.

Recently, Lagrangian measurement systems have been developed in many fields using MEMS sensor, which can measure physical quantities from the Lagrangian point of view

(following its motion) [3-6]. In the previous study, we developed a Lagrangian sensor system for force (acceleration) measurement and successfully measured the fluid force acting on a 
particle in liquid [7]. However, for a better understanding of multi-particle dynamics in a fluid flow, simultaneous measurement of the force acting on the target particle and its position (which is invisible from outside in most cases) is required.

The purpose of this study is to develop a new Lagrangian sensor system to measure both the force acting on the target object and its position non-invasively for understanding multiphase flow dynamics. Towards this end, we improved the existing sensor system developed in the previous study [7] and established a new measurement system that detects the position and the force simultaneously. We applied the new system to the floating-sinking motion of an object in a gas-solid fluidized bed, which is a kind of multiphase flow with solid particles. It is known that the gas-solid fluidized bed sometimes shows a fluid-like behavior with an apparent density. If a solid object is fed into the fluidized bed, it floats or sinks according to its density. However, the object in the fluidized bed occasionally shows unexpected floatingsinking motion and it has been reported as an interesting topic in physics [8, 9]. From the point of view of engineeing, a float-sink density separator using the fluid-like characteristics of a gas-solid fluidized bed has been developed as a dry separation technique [10]. This technique has attracted attention as a breakthrough separation method without use of water. Understanding the floating mechanism of a solid object in a fluidized bed is important for controlling the separation technique. For those reasons, we examined the vertical motion of the object in the fluidized bed, which cannot be observed from outside, by simultaneous measurement of the position and force acting on it under various conditions.

\section{LAGRANGIAN SENSOR SYSTEM}

As mentioned above, the motion of solid objects in fluid flow varies in complicated ways under the influence of the surrounding flow. In addition, the object motion is invisible in most cases of practical flow system. Therefore, direct measurements of quantities related to the object dynamics (position, velocity, etc.) are quite difficult in multiphase flows. The measurement of the motion of invisible objects from outside have been conducted, for example, utilizing non-invasive imaging techniques (X-ray imaging, Magnetic Resonance Imaging (MRI), Electrical Capacitance Tomography (ECT), Positron Emission Particle Tracking (PEPT), etc.). Some techniques have been applied to multiphase flows and granular flows $[11,12]$. In general, most of these methods are based on the technique of acquiring static 
images, and consequently they require a high-speed scanning system to detect the dynamic motions of objects. Furthermore, in order to detect the velocity and acceleration of the moving object from static images, a process for differentiating instantaneous position is required. As a result, the temporal resolution and precision of the measured data are drastically reduced.

In the previous study, we developed a non-invasive measurement system for detecting the dynamic force acting on a spherical object moving freely in a fluid flow [7, 13]. This system consists of MEMS sensors, a data processing IC and a wireless module for data transmission. Such sensors that can measure physical quantities following its motion are called Lagrangian sensors. Recently, some types of Lagrangian sensor have been proposed with the development of MEMS technology. For instance, Wadke et al. have established a Lagrangian sensor for acceleration and temperature measurements [3, 4]. Shew et al. have performed Lagrangian temperature measurement in liquid thermal convection $[5,6]$. Reinecke et al. have measured physical quantities in a stirred tank [14-16]. Moreover, Lagrangian sensors have been applied to the measurement of multiphase and granular flows [17-20]. In particular, the acceleration measurements of an object penetrated into granular media have been conducted in the field of geophysics [21-23].

However, in most cases of practical study such as modeling of fluid force in multiphase flow, both the position and force (acceleration) must be detected simultaneously to avoid the differential and integral errors in the data. Thus, we have developed a system for spontaneously measuring an object's position and acceleration by improving the previously proposed Lagrangian sensor system. The specification of the previous sensor system and the concept of the new sensor system are explained below.

Figure 1 shows the appearance of the Lagrangian sensor particle that we developed in the previous study. The built-in sensor system is composed of a 3-axis acceleration sensor (Hitachi Metals, H34C), a 3-axis magnetometer (Aichi-MI, AMI302), a microcontroller (Microchip, PIC16F877A), a wireless transmitter module and cells. The details of the characteristics and operating conditions of the sensor system can be found in the previous article [7]. The acceleration sensor, the magnetometer, and the microcontroller are deposited on both sides of a circular substrate of diameter $16 \mathrm{~mm}$. The sensor substrate and the wireless transmitter module are placed into a spherical shell made from polystyrene. The outer diameter of the shell is $d=29.8 \mathrm{~mm}$. The analog signals obtained from the built-in sensors 


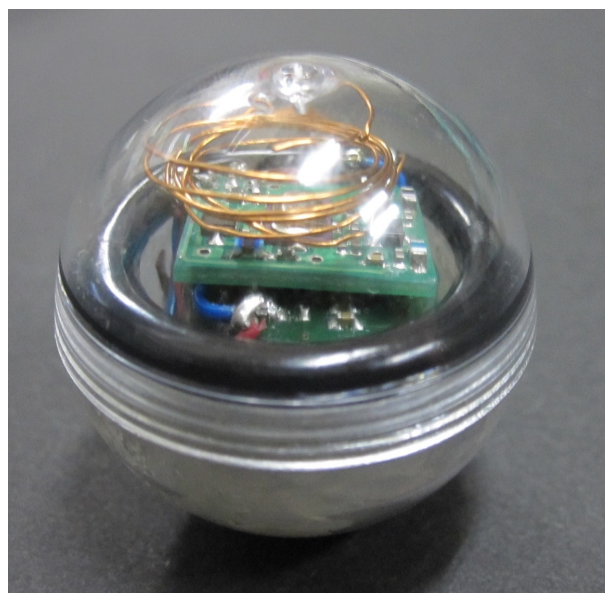

FIG. 1: Lagrangian sensor system (diameter $d=29.8 \mathrm{~mm}$ )[7].

are converted into 10bit digital signals and are serialized by USART in the microcontroller. The serialized signals are then sent to the receiving module connected to the PC wirelessly. The set of six measured data (3-axis acceleration and 3-axis magnetic vectors) is obtained every 31.25 milliseconds.

In general, a wireless data transmission is not so fast compared to other data-collection systems such as a local storage system. However, it is easy to synchronize the measured signals with other measurement data because it sends the signals in realtime. Therefore it is suited for the measurement in combination with the measurement by other sensor systems or the image acquisition by video cameras. For data transmission, we adopted a wireless module with a radio-frequency of $315 \mathrm{MHz}$. This is because we developed the Lagrangian sensor system not only for gas-solid flows but also liquid-solid flows. It is well-known that the absorption of electromagnetic wave in liquid is much more significant than that in air and greatly depends on the frequency [24]. The wireless module we used is capable of sending electromagnetic signals from a several tens of centimeter-sized water vessel to the outside [7].

The acceleration sensor we used is of a 3-axis piezo-resistive type that detects both the dynamic acceleration and the gravitational acceleration. In order to measure the force acting on a particle, only the dynamic acceleration (force divided by mass) has to be detected. However, it is difficult to get rid of the gravity and to measure only the dynamic acceleration for an arbitrary particle attitude. In order to remove the gravitational acceleration from the measured signals, we have used an external magnetic field oriented in the same 


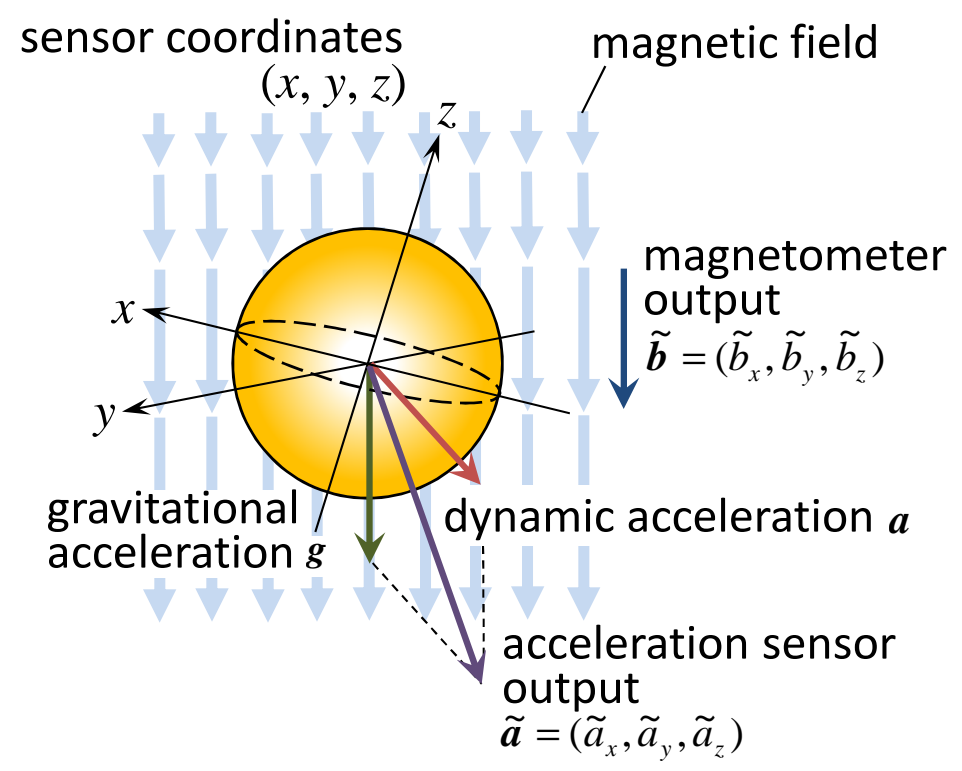

FIG. 2: Concept of spontaneous measurement for vertical position and acceleration.

direction as gravity. Figure 2 illustrates the concept of our measurement system. The 3axis magnetometer can detect the direction of the magnetic field for any particle attitude. The magnetometer outputs voltages proportional to the components of the magnetic vector $\tilde{\boldsymbol{b}}=\left(\tilde{b}_{x}, \tilde{b}_{y}, \tilde{b}_{z}\right)$ in sensor-fixed coordinates $(x, y, z)$. On the other hand, the acceleration sensor outputs the voltages proportional to the vector sum of the dynamic and the gravitational accelerations $\tilde{\boldsymbol{a}}=\left(\tilde{a}_{x}, \tilde{a}_{y}, \tilde{a}_{z}\right)$ in the sensor-fixed coordinates. Using these two vectors, the dynamic acceleration vector $\boldsymbol{a}$ is calculated by subtracting the gravity from the output of the acceleration sensor as

$$
\boldsymbol{a}=\tilde{\boldsymbol{a}}-g \frac{\tilde{\boldsymbol{b}}}{|\tilde{\boldsymbol{b}}|}
$$

where $g$ is the constant of gravitational acceleration $\left(g=9.81 \mathrm{~m}^{2} / \mathrm{s}\right)$. The acceleration vector given by Eq.(1) can be obtained as the components in the sensor-fixed coordinates, i.e., $\boldsymbol{a}=\left(a_{x}, a_{y}, a_{z}\right)$. By calculating the dot product of $\boldsymbol{a}$ with the unit vector in the gravitational direction $\tilde{\boldsymbol{b}} /|\tilde{\boldsymbol{b}}|$, the vertical component $a_{V}$ is obtained as follows.

$$
a_{V}=\boldsymbol{a} \cdot \frac{\tilde{\boldsymbol{b}}}{|\tilde{\boldsymbol{b}}|}-g
$$

As described in our article [7], the accuracy of vertical acceleration (vertical force) is less influenced by the magnetic linearity (inclination of magnetic field) because the error of the magnetic vector (inclined angle $\Delta \theta$ ) reveals the final acceleration output to be $\cos \Delta \theta$, which is a slowly varying function around zero. 


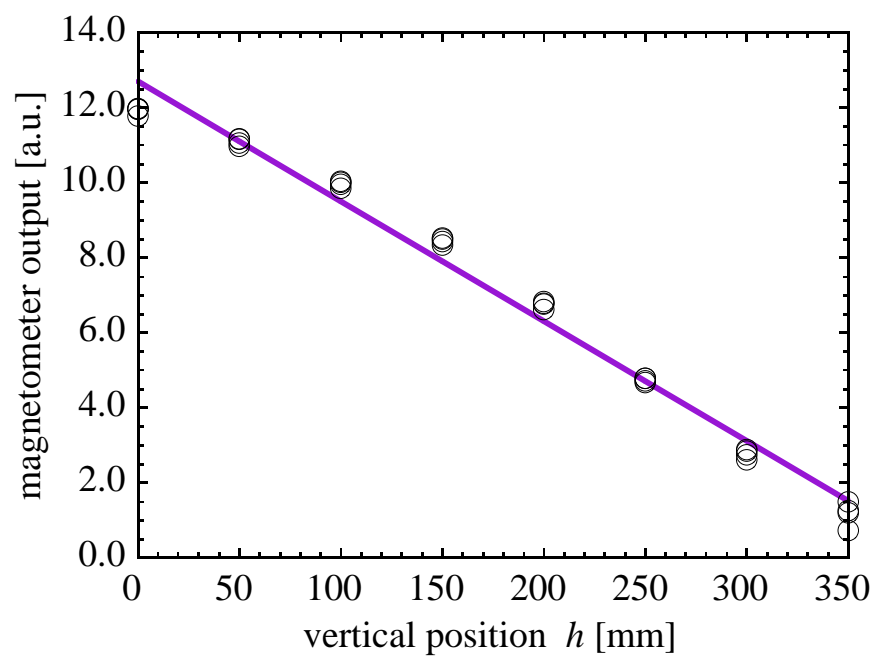

FIG. 3: Relation between vertical position and magnetometer output (standard curve for position measurement).

By the procedure described above, the vertical force acting on the object (Lagrangian sensor) can be detected by removing the gravitational acceleration from the acceleration output with the help of external magnetic field. However, it is found from Eq.(2) that only the direction of the magnetic vector $\tilde{\boldsymbol{b}} /|\tilde{\boldsymbol{b}}|$ is used and its magnitude $|\tilde{\boldsymbol{b}}|$ is discarded in the procedure. Therefore we make use of this information to detect the vertical position of the object. We use a gradient coil system (Maxwell coil) as a source of the external magnetic field in the new experimental system (See Fig.7). The Maxwell coil consists of two identical coils located with the same centerline. As is well-known, the Maxwell coil produces a linear magnetic field with a uniform gradient at the centerline as follows.

$$
B(z)=\frac{\mu_{0} N I R^{2}}{\left(R^{2}+z^{2}\right)^{3 / 2}}
$$

where $B$ is the magnetic field, $I$ is the current, $R$ is the coil radius, $\mu_{0}$ is the permeability constant and $N$ is the turn number of each coil. If the Maxwell coil is used in our Lagrangian sensor system, we can detect the vertical position $z$ from the magnitude of the magnetic vector $B(z)$, which was discarded in the previous measurement system.

Based on Eq.(3), we created a magnetic field using two coils with $R=400 \mathrm{~mm}$ and $N=176$. The distance between the two coils is $\sqrt{3} R=693 \mathrm{~mm}$. The DC power supply is used and the current is controlled by variable resistors. We use only the lower part of the inner region of the coil system where the magnetic vector is directed downward. In addition to the main coil system, a supplementary coil system is set horizontally to cancel 
the terrestrial magnetic field. The appearance of the coil system is shown later (Fig.6 and Fig.7).

Figure 3 shows the relationship between the vertical position and the magnetometer output obtained from a calibration experiment. We measured the vertical component of the magnetic vector at five points in each cross-section, i.e., the central point and the four adjacent points $( \pm 60 \mathrm{~mm}$ apart from the center). As can be seen in Fig.3, the magnitude of the magnetic vector is almost constant in each cross-section and changes linearly with height. From these results, the standard curve (solid line in Fig.3) which relates the vertical position of the Lagrangian sensor particle to the magnetometer output is obtained.

The straightness of the magnetic vector almost holds in the inner region of the coil system. The angle of the magnetic vector relative to the vertical line is within $3^{\circ}$ along the center line and within $10^{\circ}$ in most points even at the outer points of $60 \mathrm{~mm}$ square. However, the inclination of the magnetic vector shows a larger value (maximum $14.6^{\circ}$ ) especially at the edge of the measured region. As mentioned above, the error caused by the inclination of the magnetic field affects the acceleration (force) measurement as a cosine of the angle. For example, the inclination of $10^{\circ}$ produces $1.6 \%$ error in the measurement of vertical force because $\cos 10^{\circ}=0.984$ [7]. From these considerations, the accuracy of the vertical force measurement still holds even in the new system using the gradient magnetic field. However, as described in the previous article [7], the measurement of horizontal force (acceleration) by the present system has low accuracy. Therefore we measured only the vertical force and vertical position of the object in this study.

\section{PRELIMINARY EXPERIMENT}

The accuracy of the force measurement using our Lagrangian system has already checked in the previous article [7]. In order to check the applicability of the new Lagrangian sensor system to the simultaneous measurement of the vertical position and force, the preliminary experiment (the harmonic-vibration experiment) was performed. A spring (spring constant $k=1.18 \mathrm{~N} / \mathrm{m}$ ) was put on the upper side of Lagrangian sensor and used to measure the force (acceleration) and the position of the particle during vertical vibration. The mass of the sensor particle was controlled using clay paste and lead pieces and was set to $m=0.03$ $\mathrm{kg}$. 


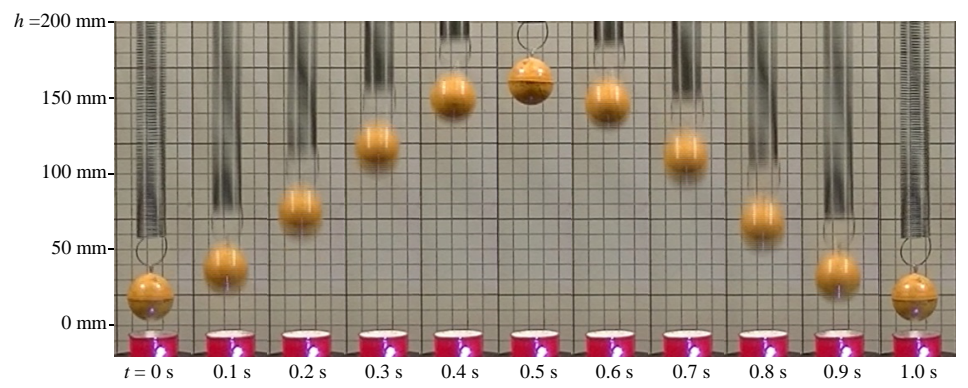

FIG. 4: Particle motion in spring experiment for one period.

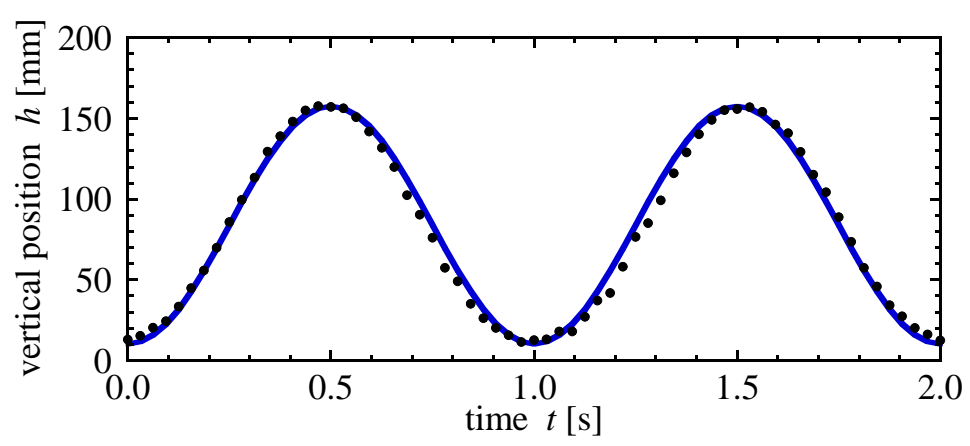

(a) vertical position

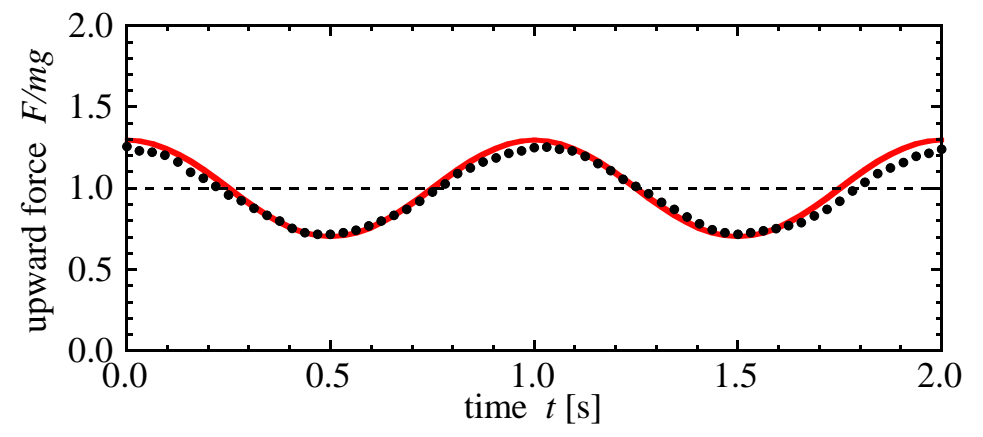

(b) upward force

FIG. 5: Vertical position and upward force (acceleration) measured by Lagrangian sensor particle.

Figure 4 shows successive pictures of the harmonic-vibration experiment. The amplitude of harmonic vibration is $A=73.7 \mathrm{~mm}$. The corresponding angular velocity and vibration period are $\omega=\sqrt{k / m}=6.27 \mathrm{rad} / \mathrm{s}$ and $T=2 \pi / \omega=1.00 \mathrm{~s}$ respectively. Figures 5 (a) and (b) indicate the vertical position $h$ and the normalized upward force $F / m g$ (or normalized vertical acceleration $a / g$ ) obtained from non-invasive measurement by the Lagrangian sensor. The solid lines in the figures indicate the position $A \sin \omega t$ and the acceleration $-A \omega^{2} \sin \omega t$ obtained theoretically. The measured position and acceleration are in good agreement with theory. Although all the possible errors are not evaluated by the vibration experiment, at least, it can be said that the simultaneous measurement of the vertical position and the 
vertical force acting on the object can be achieved by the new Lagrangian sensor system.

\section{EXPERIMENTAL SETUP OF A GAS-SOLID FLUIDIZED BED}

The new Lagrangian measurement system is applied to the floating-sinking motion of a large object in a gas-solid fluidized bed. Figures 6 and 7 indicate the schematic diagram and the picture of experimental apparatus, respectively. The experimental system is composed of an air-inlet system (blower, valve, etc.), a rectangular vessel for the fluidized bed, a Maxwell coil system, a Lagrangian sensor particle, a wireless data-receiving module and a personal computer. The vessel for the fluidized bed has a $150 \mathrm{~mm}$ square cross-section. The distributor installed at the bottom of the vessel has a multilayer structure made of SUS mesh films, sponges, and filter clothes. The height of the coil system is adjusted so that the lower coil is at the same height as the bottom of the fluidized region $(h=0 \mathrm{~mm})$.

The fluidized particles are glass beads with diameter $d_{p}=600-850 \mu \mathrm{m}$ and density $\rho_{p}=2.5 \mathrm{~g} / \mathrm{cm}^{3}$. The inlet air velocity $u_{0}$ is controlled with measuring the air mass flow rate by a mass flow controller and it is set based on the minimum fluidization velocity $u_{\mathrm{mf}}$ as $u_{0} / u_{\mathrm{mf}}=1.5,2.0,3.0,4.0,5.0,6.0$ and 7.0 . In order to examine the floating-sinking motion by the "hydrostatic effect", the density of the Lagrangian sensor $\rho_{s}$ is controlled. As explained below, if the fluidization of particles is uniform, the mass of fluidized particles must be supported by air and then the linear pressure profile like hydrostatic pressure is generated in the bed. As a result, the "buoyancy force" is acting on the object immersed in the fluidized bed. The mass of the Lagrangian sensor is adjusted by putting clay paste and lead pieces inside. The density of the Lagrangian sensor is set as $\rho_{s}=0.9,2.1$ and $2.35 \mathrm{~g} / \mathrm{cm}^{3}$. The center of the gravity of the sensor is not located at the center of the spherical capsule precisely. However, we adjusted the mass distribution in the capsule to prevent the unnatural motion of the Lagrangian sensor caused by the bias of the mass.

Before starting the air flow, we put fluidized particles (glass beads) in the experimental vessel uniformly. The initial bed height is $L=150 \mathrm{~mm}$. Then the Lagrangian sensor particle is put at the center of the bed surface. After checking that the signal is transmitted from the sensor, the air supply is started. The motions of the fluidized particles and the Lagrangian sensor are recorded by a digital video camera system synchronized with data acquisition of the Lagrangian sensor output. The Lagrangian measurement is performed for 70 seconds. 


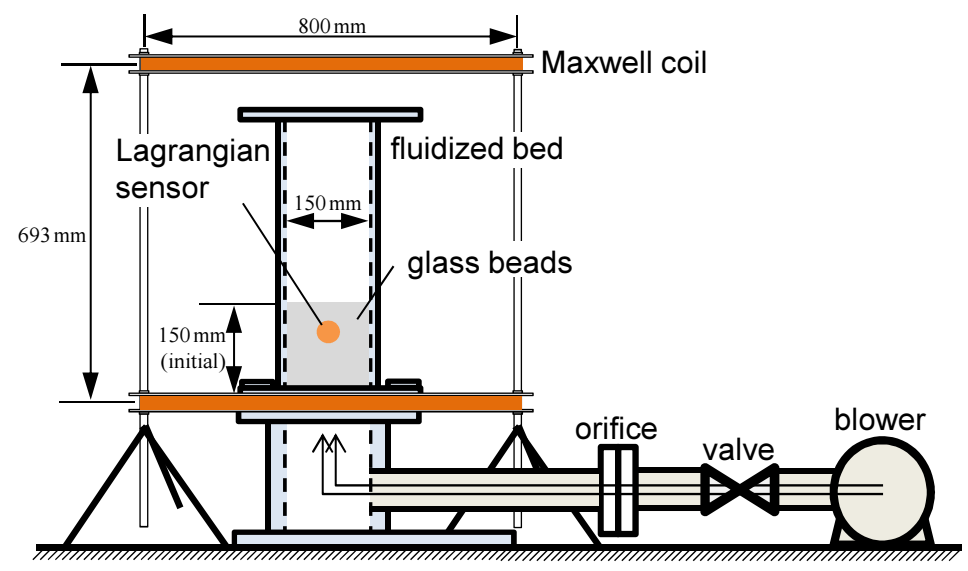

FIG. 6: Schematic diagram of experimental apparatus of gas-solid fluidized bed.

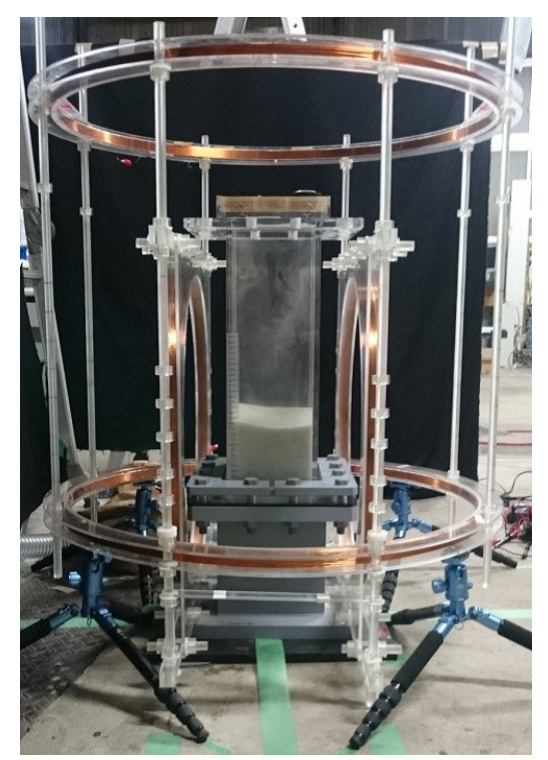

FIG. 7: Appearance of gas-solid fluidized bed and Maxwell coil system.

Hereafter we pick up the measured data from 30 to 60 seconds for discussion because the fluidization reaches a fully steady state after 30 seconds of air supply.

\section{RESULTS AND DISCUSSION}

Figure 8 shows the flow pattern of gas-solid fluidized bed under various air-velocity conditions. For low velocity $\left(u_{0} / u_{\mathrm{mf}}=1.5-3.0\right)$, the flow pattern shows "bubbling-fluidization", i.e., the bed fluidizes almost uniformly and bubbles sometime rise up in the bed [25]. On the other hand, the flow pattern becomes more complicated and changes to "turbulent 


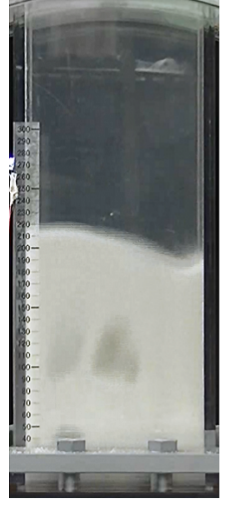

(a) 1.5

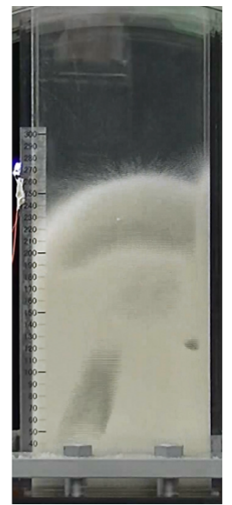

(b) 2.0

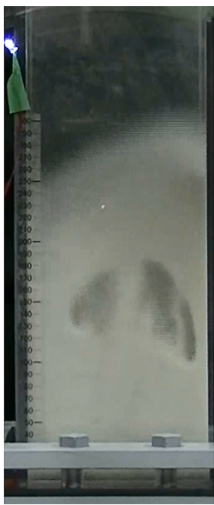

(c) 3.0

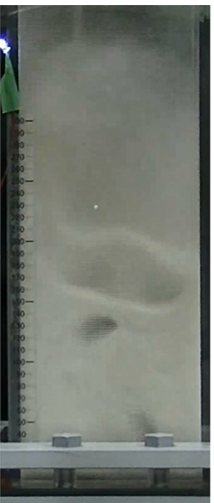

(d) 4.0

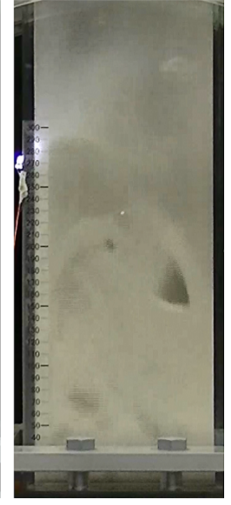

(e) 5.0

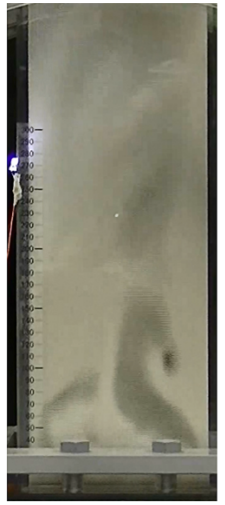

(f) 6.0

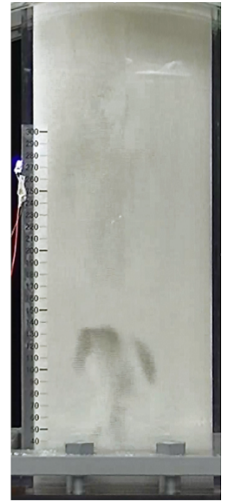

(g) 7.0

FIG. 8: Flow pattern of gas-solid fluidized bed (values under each picture indicate $u_{0} / u_{\mathrm{mf}}$ ).

fluidization" with the increase of air velocity $\left(u_{0} / u_{\mathrm{mf}}=4.0-7.0\right)$. It is also found that the average bed height increases with increasing air velocity. The bed height with low air velocity $\left(u_{0} / u_{\mathrm{mf}}=1.5\right)$ does not change so much from the initial bed height, while it drastically changes in case of high air velocity. If the fluidization is uniform and the bed height is constant, the pressure gradient in the bed is expressed as follows.

$$
\Delta p / L=\rho_{a} g
$$

where $\rho_{a}=\rho_{p}(1-\epsilon)(\epsilon$ : void fraction $)$ is the apparent particle density in fluidization. We estimate the apparent density from the mass of particles and the initial bed height to be about $1.5 \mathrm{~g} / \mathrm{cm}^{3}$ in this experimental condition. This value roughly indicates the float $/ \mathrm{sink}$ motion of a large object put in the fluidized bed. If the density of the object is smaller/larger than the apparent density of fluidized particles, it basically floats/sinks in the bed due to the "buoyancy force".

Here we present the typical behaviors of the object (Lagrangian sensor particle) in the gas-solid fluidized bed. Figure 9 indicates measured vertical position of the object in three typical modes, (a) floating mode $\left(\rho_{s}=0.9 \mathrm{~g} / \mathrm{cm}^{3}\right.$ and $\left.u_{0} / u_{\mathrm{mf}}=1.5\right)$, (b) sinking mode $\left(\rho_{s}=\right.$ $2.35 \mathrm{~g} / \mathrm{cm}^{3}$ and $\left.u_{0} / u_{\mathrm{mf}}=4.0\right)$ and (c) floating-sinking mode $\left(\rho_{s}=2.1 \mathrm{~g} / \mathrm{cm}^{3}\right.$ and $\left.u_{0} / u_{\mathrm{mf}}=1.5\right)$. As mentioned above, the apparent density of fluidized particles is $\rho_{a}=1.5 \mathrm{~g} / \mathrm{cm}^{3}$. Basically, an object with a density lower than the apparent density will float in the fluidized bed. Fig.9 (a) indicates that the light object $\left(\rho_{s}=0.9 \mathrm{~g} / \mathrm{cm}^{3}\right)$ rises up/falls down quasi-periodically around a height $h=150 \mathrm{~mm}$. As explained later, this means that the object always exists at the free surface of the fluidized bed and moves with the surface flapping. On the other 


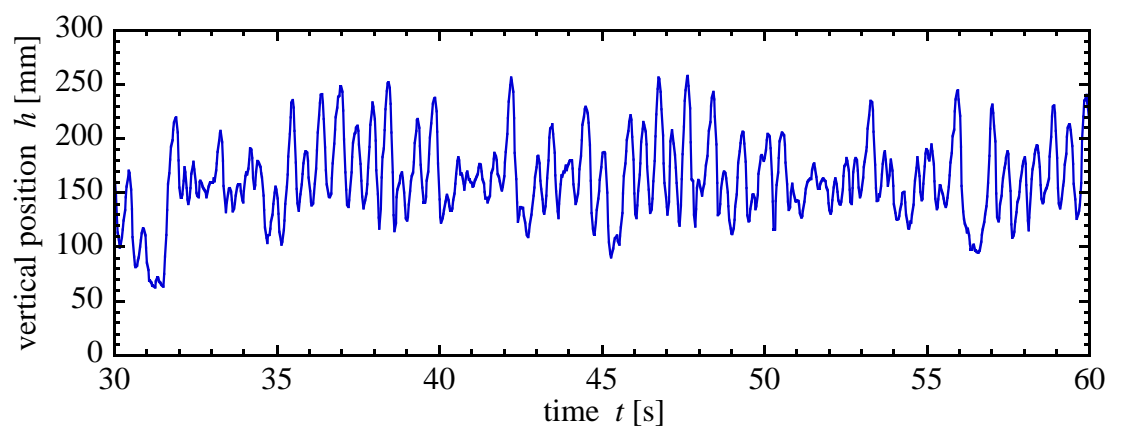

(a) floating mode $\left(\rho_{s}=0.9 \mathrm{~g} / \mathrm{cm}^{3}, u_{0} / u_{\mathrm{mf}}=1.5\right)$

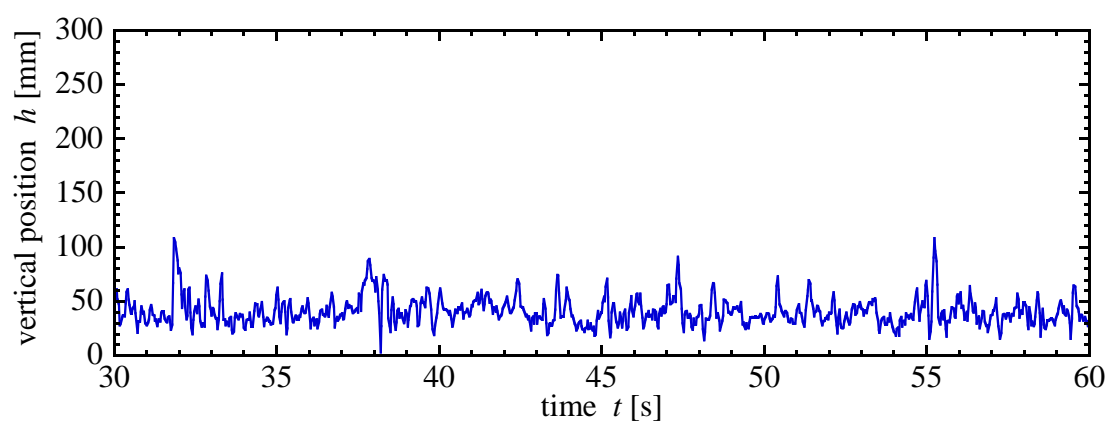

(b) sinking mode $\left(\rho_{s}=2.35 \mathrm{~g} / \mathrm{cm}^{3}, u_{0} / u_{\mathrm{mf}}=4.0\right)$

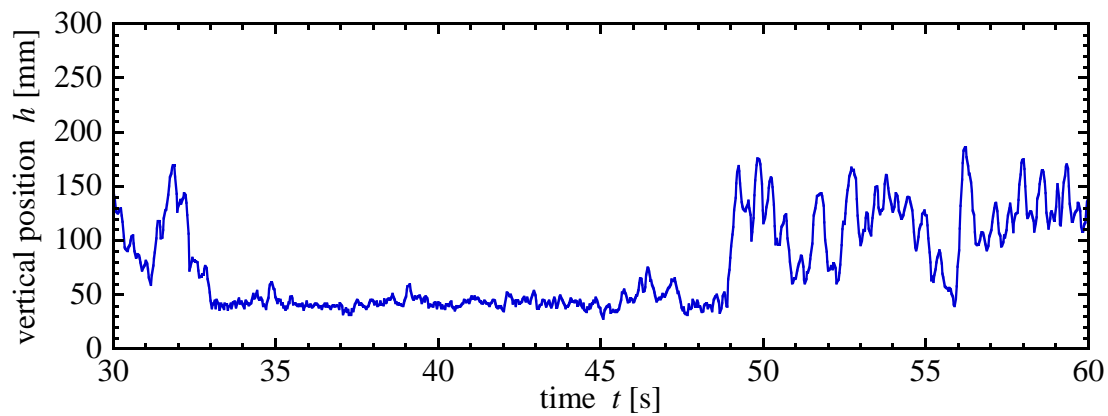

(c) floating-sinking mode $\left(\rho_{s}=2.1 \mathrm{~g} / \mathrm{cm}^{3}, u_{0} / u_{\mathrm{mf}}=1.5\right)$

FIG. 9: Instantaneous vertical position of Lagrangian particle in gas-solid fluidized bed at steady state $(t=30-60 \mathrm{~s})$.

hand, the heavy object $\left(\rho_{s}=2.35 \mathrm{~g} / \mathrm{cm}^{3}\right)$ sinks in the bed and trembles near the bottom (Fig.9b). These behaviors of light and heavy objects can be explained by hydrostatic effects as mentioned above. However, the object with a density close to the apparent density of the fluidized bed $\left(\rho_{s}=2.1 \mathrm{~g} / \mathrm{cm}^{3}\right)$ shows strange motion, i.e., it keeps floating or sinking in turn for the period of several tens of seconds (Fig.9c). These results indicate that the floatingsinking motion of objects in gas-solid fluidized bed cannot be explained by hydrostatic effects alone, i.e., it is not determined only by the object density as reported in the previous study [8]. 

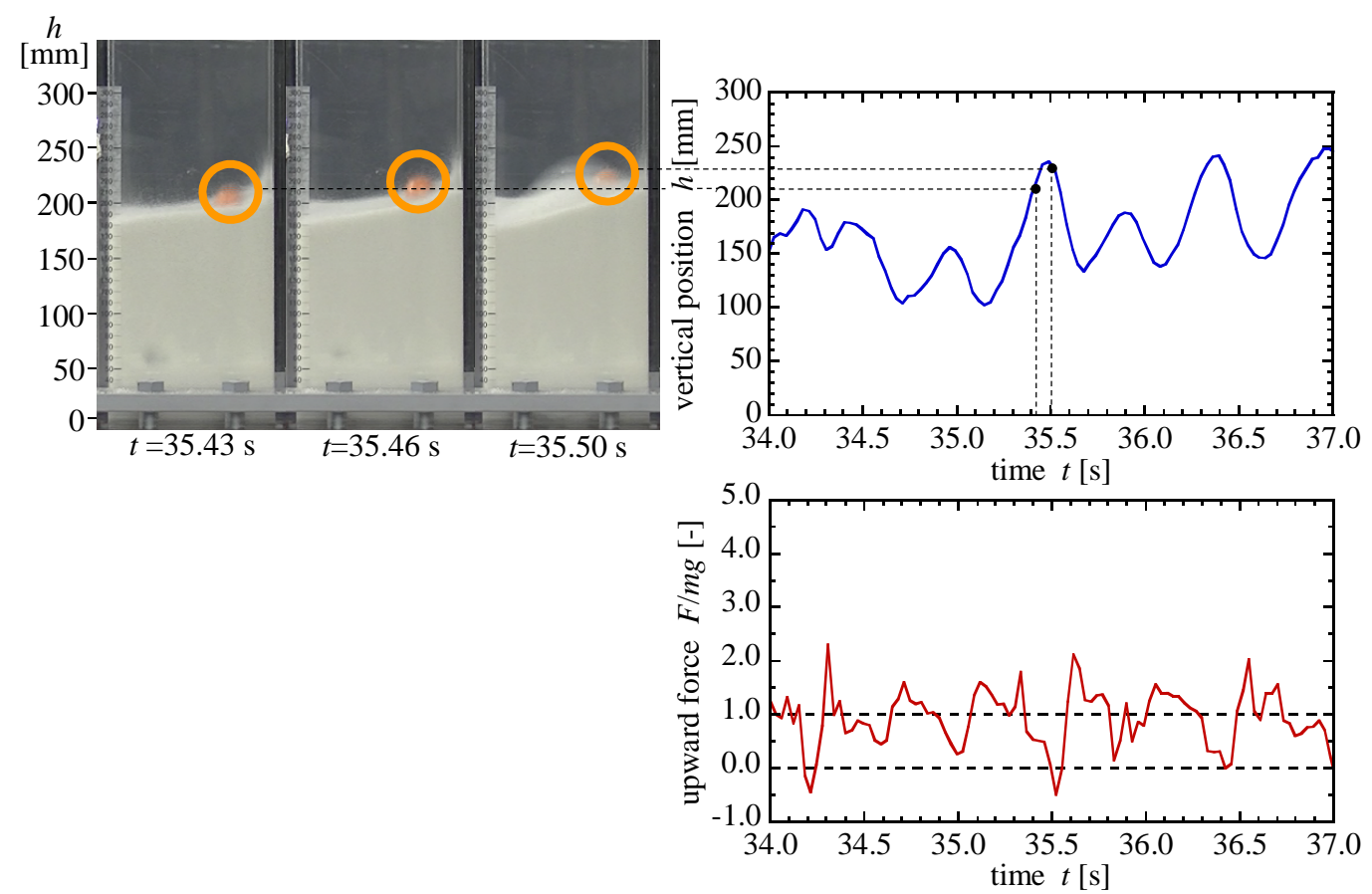

FIG. 10: Vertical position and upward force acting on Lagrangian particle in gas-solid fluidized bed in floating mode $\left(\rho_{s}=0.9 \mathrm{~g} / \mathrm{cm}^{3}, u_{0} / u_{\mathrm{mf}}=1.5\right)$.

In order to examine the details of the object motion in the fluidized bed, we focus on measurement results over a short period. Figure 10 indicates successive pictures of the fluidized bed as well as measurement results for the position and upward force acting on the object in the floating mode. The pictures in Fig.10 are selected from those in which the position of the object (Lagrangian sensor particle) can be recognized from outside. The measurement results of the position are in good agreement with the observation results. It can be confirmed that our measurement system can detect changes in the vertical position of the object for several tens of milliseconds.

In Fig.10, measurement results of the upward force are normalized by the gravity force. The normalized force becomes unity if the upward force acting on the object is balanced with the gravity force (stationary state or uniform motion). On the other hand, if no upward force is acting on the object (free-falling state), the normalized force indicates zero. By comparison with the vertical position and the upward force, the motion of the object in the fluidized bed can be understood distinctly. If we put a light object in a fluidized bed with low air velocity, the object floats at the free surface of the bed by the hydrostatic effect. As shown in Fig.8, the flow pattern of the fluidized bed with air velocity $u_{0} / u_{\mathrm{mf}}=1.5$ is a "bubbling-fluidized" 
state and isolated bubbles rise up intermittently. From the observation of the fluidized state, the frequency of a bubble generation in the fluidized bed is roughly estimated as $2 \mathrm{~Hz}$. It is found from Fig.10 that the object moves vertically in synchronization with the bubble motion obviously. The upward force acting on the object also varies periodically and it shows a " $\gamma$ "-shaped change. In our previous study [13], the floating motion of the object is characterized by three stages. In the first stage, the bed expands due to a rising bubble and the floating object moves upward with the motion of the free surface. At this moment, the upward force acting on the object is almost zero because the object motion is slow (the first flat of " $\gamma$ "). In the next stage, the rising bubble reaches the free surface and it collapses. The object falls into a crater by the gravity and in consequence the upward force rapidly approaches zero (the successive slope of " $\gamma$ "). Finally, the object collides with the bottom of the crater and an impulsive upward force is exerted on it (the vertical part of " $\gamma$ ").

The upward force acting on the object is obviously the combination of the contact force with fluidized particles and the fluid force in this case. In our previous study, the contribution of the contact and fluid forces acting on the object in the fluidized bed was examined by discrete particle simulation $[13,26]$. The simulation results indicated that the fluid force is much more significant than the contact force except for the final impulsive stage. Surprisingly, the object is supported not by surrounding particles but by air in most of the floating motion period. Therefore, the object does not float in the surrounding particles, but the object and the surrounding particles do float together in air, even in the floating mode shown in Fig.10.

Next we discuss the motion of a heavy object in the fluidized bed. Figure 11 shows the vertical position of the heavy object and the upward force acting on it $\left(\rho_{s}=2.35 \mathrm{~g} / \mathrm{cm}^{3}\right)$ when it is placed in the fluidized bed with moderate air velocity $\left(u_{0} / u_{\mathrm{mf}}=4.0\right)$. The flow pattern of the fluidized bed is a "bubbling-turbulent" state in which many small bubbles rise up in the bed irregularly. As shown in the figure, the object sinks into the bottom of the bed immediately. The object motion can be basically explained in that, since the density of the object is larger than the apparent density of fluidized particles, the gravity force exceeds the "buoyancy force" in the bed and consequently the object remains at the bottom. However, the measured upward force fluctuates around unity and sometimes a large upward force is exerted on the object. In the fluidized bed with moderate air velocity, many small bubbles are observed to generate constantly from the distributor. As a result, a thin void layer is 

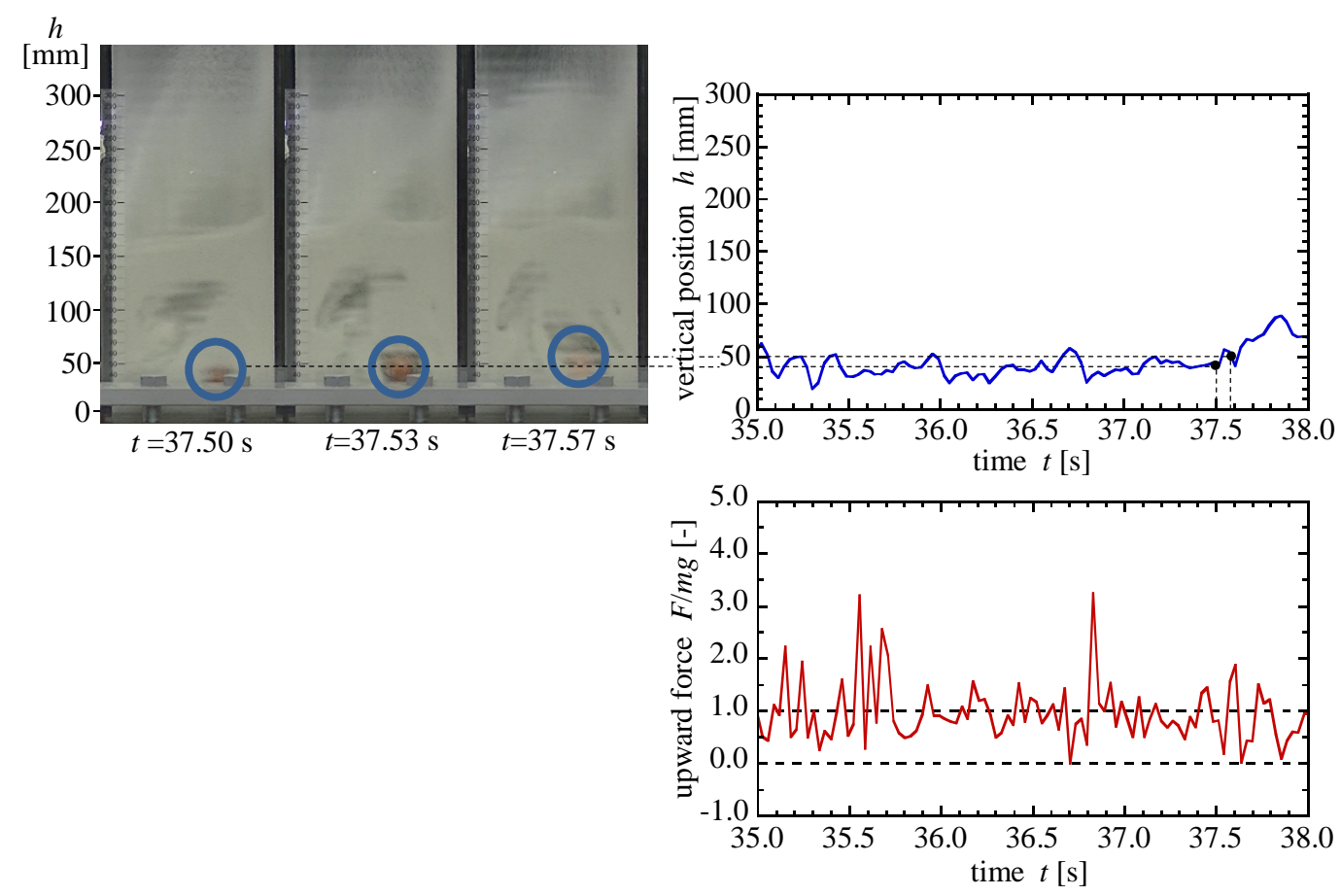

FIG. 11: Vertical position and upward force acting on Lagrangian particle in gas-solid fluidized bed in sinking mode $\left(\rho_{s}=2.35 \mathrm{~g} / \mathrm{cm}^{3}, u_{0} / u_{\mathrm{mf}}=4.0\right)$.

formed near the bottom of the bed. The fluctuation of the position and the force shown in Fig.11 indicate that the object is trapped in the void layer and struggles to escape from there. These facts suggest the existence of another lifting mechanism in addition to the buoyancy effect in the gas-solid fluidized bed.

The motion of the object with moderate density is also interesting. Figure 12 indicates the vertical position and upward force acting on the object with a density of $\rho_{s}=2.1 \mathrm{~g} / \mathrm{cm}^{3}$ in the fluidized bed with small air velocity $\left(u_{0} / u_{\mathrm{mf}}=1.5\right)$. The flow pattern of the fluidized bed is a "bubbling" state, i.e., the fluidization is almost uniform and a large bubble rises up intermittently. Since the density of the object is larger than the apparent density of the fluidized particles, it should basically sink into the bottom of the bed. It is found from Fig.12 that the object go up and down in the middle of the bed repeatedly. However, it should be noted that the results shown here is a part of the long-time behavior shown in Fig.9(c). Under this condition, the object remains at the bottom or floats in the bed by turns. In other words, the object is sometimes trapped in the void layer at the bottom of the bed and it occasionally succeeds in escaping from there. During the floating period, the object exerts a " $\gamma$ "-shaped periodic force synchronized with up/down motion (Fig.12). 

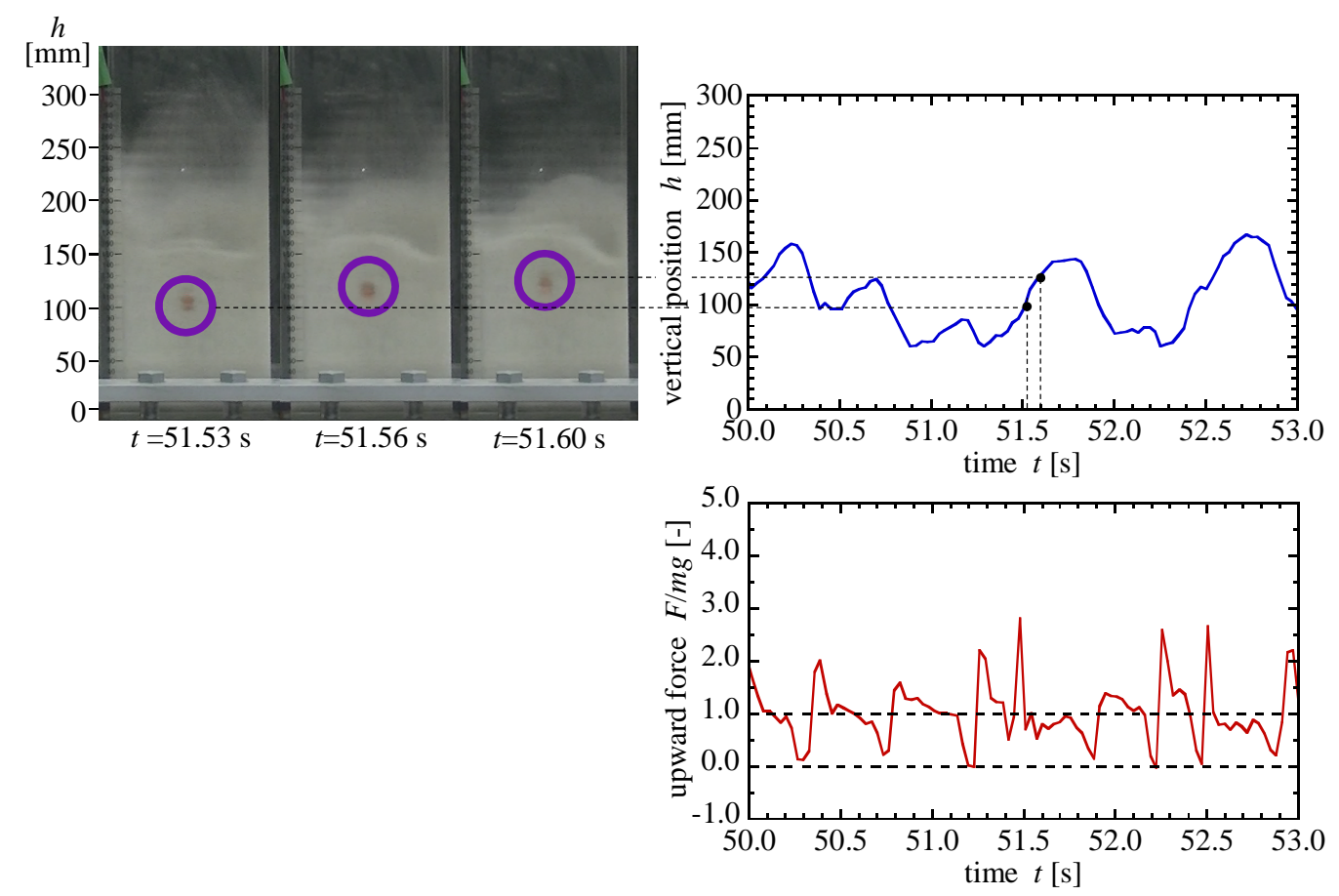

FIG. 12: Vertical position and upward force acting on Lagrangian particle in gas-solid fluidized bed in floating-sinking mode $\left(\rho_{s}=2.1 \mathrm{~g} / \mathrm{cm}^{3}, u_{0} / u_{\mathrm{mf}}=1.5\right)$.

This suggests that the object always interact with rising bubbles even when floating in the fluidized bed.

To summarize, the floating-sinking motion of a large object in a gas-solid fluidized bed can be basically explained by the buoyancy effect, which results from "hydrostatic pressure" formed in the bed. Meanwhile, there is another floating/sinking mechanism arising from inhomogeneous and unsteady fluidization such as bubbles and void layers in the bed.

Figure 13 shows the probability density of the vertical position under various conditions of object density and air velocity. The probability density of each condition is calculated from the measurement data of vertical position for 30 seconds. As shown in the figure, the profiles of the probability density are clearly classified into three patterns. i.e., the broad profile throughout the bed, the sharp profile at the bottom of the bed and the superposition of these profiles. From these profiles, we can separate the object motion in the fluidized bed into three modes as floating (F), sinking (S), and floating-sinking (FS) intuitively. The floating mode can be seen in cases of small object density $\left(\rho_{s}=0.9 \mathrm{~g} / \mathrm{cm}^{3}\right)$, regardless of air velocity. In this mode, the object follows the motion of the free surface of the fluidized bed which flaps around the height $h=150 \mathrm{~mm}$. This is consistent with hydrostatic concept, 


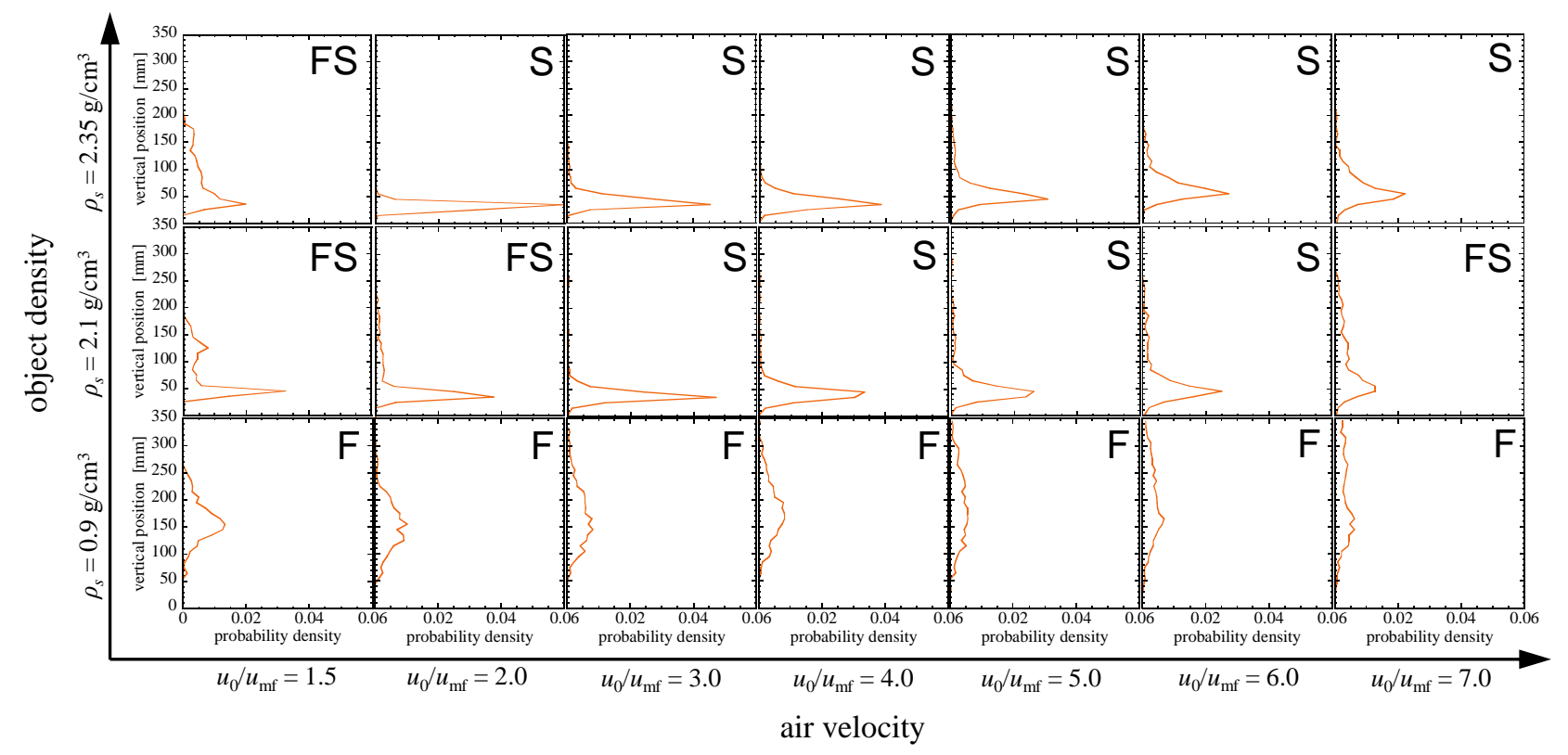

FIG. 13: probability density of vertical position under various conditions of object density and air velocity for 30 seconds.

i.e., that the object floats if its density is smaller than the apparent density of the fluidized bed $\left(\rho_{a}=1.5 \mathrm{~g} / \mathrm{cm}^{3}\right)$. It can also be found from the results for the floating mode that the probability density distribution is broaden with the increase of air velocity. This is because the flow pattern of the fluidized bed with high air velocity changes into a turbulent state and the free surface moves violently.

The sinking mode can be found in most cases of heavy objects $\left(\rho_{s}=2.1 \mathrm{~g} / \mathrm{cm}^{3}\right.$ and $\left.2.35 \mathrm{~g} / \mathrm{cm}^{3}\right)$. In this mode, the object sinks in the fluidized bed due to the density difference and is the trapped in the void layer, which is a dilute region of fluidized particles. From Fig.13, it can be seen that the object is located in a certain range at the bottom of the bed. This region corresponds to the void layer and grows wider with higher air velocity.

It is interesting to note that the floating-sinking mode can be seen in the motion of the object with moderate density under the conditions of both small and large air velocity. In this mode, the object is trapped in the void layer for a certain period and occasionally floats into the upper fluidized region. As mentioned above, local inhomogeneity of the fluidization of particles may affect the motion of the object in the fluidized bed. Therefore, the floating-sinking behavior of the object is determined not only by its density but also by 
local fluidization state in the fluidized bed.

\section{CONCLUSIONS}

A Lagrangian measurement technique for the position and the acceleration (force) of a solid object has been developed. The measurement system, which is composed of a wireless sensor system and a Maxwell coil system, can detect both the vertical position and the vertical force acting on the object non-invasively. The developed system was applied to the floating-sinking motion of a large object in a gas-solid fluidized bed which is invisible from outside. The measured results showed that the motion of a large object is influenced not only by the hydrostatic effect (buoyancy effect) of gas-solid fluidized bed but also by local variance of the fluidization state of particles.

The measurement system proposed in this study has some advantages compared to existing systems. Our system can measure the vertical position and acceleration simultaneously. Therefore it is possible to calculate the velocity with high accuracy from both measurement data by avoiding differential and integral errors. Also it can measure the motion of an object in water, as shown in the previous study[7]. The largest advantage of our system is the cost. We manufactured the system for only a few thousand US dollars, which is obviously cheaper than other position detecting systems based on non-invasive imaging techniques. 
[1] Brennen, C. E., Fundamentals of multiphase flows, Cambridge University Press, Cambridge (2005).

[2] Clift, R., Grace, J. R. and Weber, M. E.,Bubbles, drops and particles, Dover Pub. Inc., Mineola (1978).

[3] Wadke, P. M., Hounslow, M. J. and Salman A. D., The Smart sphere: Experimental results, Chem. Eng. Res. Des., 83 (2005) 1298-1302.

[4] Wadke, P. M., Salman, A. D. and Hounslow, M. J., The Smart temperature sphere: Application in rotary drum mixers, Pow. Tech., 185 (2008) 274-279.

[5] Shew, W. L., Gasteuil, Y., Gibert, M., Metz, P. and Pinton J.-F., Instrumented tracer for Lagrangian measurements in Rayleigh-Bnard convection, Rev. Sci. Instrum., 78 (2007) 065165.

[6] Gasteuil, Y., Shew, W. L., Gibert, M., Chillá, F., Castaing, B. and Pinton, J.-F., Lagrangian temperature, velocity, and local heat flux measurement in Rayleigh-Bénard convection, Phys. Rev. Lett., 99 (2007) 234302.

[7] Harada, S., Kobayashi, Y., Sawano, T. and Noguchi, E., Direct measurement of fluid force on a particle in liquid by telemetry system, Int. J. Multiphase Flow, 37 (2011) 898-905.

[8] Oshitani, J., Sasaki, T., Tsuji, T., Higashida, K. and Chan, D. Y. C., Anomalous sinking of spheres due to local fluidization of apparently fixed powder beds, Phys. Rev. Lett., 116 (2016) 068001.

[9] Penn, A., Tsuji, T., Brunner, D. O., Boyce, C. M., Pruessmann, K. P. and Müller, C. R., Realtime probing of granular dynamics with magnetic resonance, Sci. Adv., 3 (2017) e1701879.

[10] Oshitani, J., Kajimoto, S., Yoshida, M., Franks G. V., Kubo, Y. and Nakatsukasa, S., Continuous float-sink density separation of lump iron ore using a dry sand fluidized bed dense medium, Adv. Pow. Tech. 24 (2013) 468-472.

[11] Kawaguchi, T., MRI measurement of granular flows and fluid-particle flows, Adv. Powder Technol., 21 (2010) 235-241

[12] Sun, J. and Yan, Y., Non-intrusive measurement and hydrodynamics characterization of gassolid fluidized beds: A review, Meas. Sci. Technol., 27 (2016) 112001.

[13] Higashida, K., Rai, K., Yoshimori, W., Ikegai, T., Tsuji, T., Harada, S., Oshitani, J. and Tanaka, T., Dynamic vertical forces working on a large object floating in gas-fluidized bed: 
Discrete particle simulation and Lagrangian measurement, Chem. Eng. Sci., 151, (2016) 105115.

[14] Thiele, S., Silva, J. D. and Hampel, U., Autonomous sensor particle for parameter tracking in large vessels, Meas. Sci. Technol., 21 (2010), 085201.

[15] Reinecke, S. F., Deutschmann, A., Jobst, K., Kryk, H., Friedrich, E. and Hampel, U., Flow following sensor particlesValidation and macro-mixing analysis in a stirred fermentation vessel with a highly viscous substrate, Biochem. Eng. J., 69 (2014) 159-171.

[16] Reinecke, S. F., Deutschmann, A., Jobst, K. and Hampel, U., Macro-mixing characterisation of a stirred model fermenter of non-Newtonian liquid by flow following sensor particles and ERT, Chem. Eng. Res. Des., 118 (2017) 1-11.

[17] Köhler, A., Rasch, A., Pallarés and Johnsson, F., Experimental characterization of axial fuel mixing in fluidized beds by magnetic particle tracking, Powder Technol., 316 (2017) 492-499.

[18] Akeila, E., Salcic Z. and Swain, A., Smart pebble for monitoring riverbed sediment transport, IEEE. Sens. J., 10 (2010) 1705-1717.

[19] Neuwirth, J., Antonyuk, S.. Heinrich, S. and Jacob, M., Magnetic particle tracking for nonspherical particles in a cylindrical fluidized bed, Chem. Eng. Sci., 21 (2013) 151-163.

[20] Sánchez-Colina, G., Alonso-Llanes, L., Martínez, E., Batista-Leyva, A.J., Clement, C., Fliedner, C., Toussaint, R. and Altshuler, E., Note: Lock-in accelerometry to follow sink dynamics in shaken granular matter, Rev. Sci. Instrum., 85 (2014) 126101.

[21] Altshuler, E., Torres, H., González-Pita, A., Sánchez-Colina, G., Pérez-Penichet, C., Waitukaitis, S. and Hidalgo, R. C., Settling into dry granularmedia in different gravities, Geophys. Res. Lett., 41 (2014) 3032-3037.

[22] Sunday, C., Murdoch, N., Cherrier, O., Morales Serrano, S., Valeria Nardi, C., Janin, T., Avila Martinez, I., Gourinat, Y. and Mimoun, D., A novel facility for reduced-gravity testing: A setup for studying low-velocity collisions into granular surfaces, Rev. Sci. Instrum., 87 (2016) 084504 .

[23] Caviezel, A. and Gerber, W., Brief Communication: Measuring rock decelerations and rotation changes during short-duration ground impacts, Nat. Hazards Earth Syst. Sci., 18 (2018) 31453151.

[24] Jackson, J. D., Classical Electrodynamics (3rd Ed.), John Wiley \& Sons, Inc. (1999).

[25] Geldart, D., Types of gas fluidization, Powder Technol., 7 (1973) 285-292. 
[26] Tsuji, T., Higashida, K., Okuyama, Y. and Tanaka, T., Fictitious particle method: A numerical model for flows including dense solids with large size difference, AIChE J., 60 (2014) 1606-1620. 